\title{
Prevalencia de infección nosocomial en hospitales universitarios de La Habana, Cuba
}

\author{
H. GUANCHE GARCELL ${ }^{1}$, L. NÚÑEZ LABRADOR ${ }^{2}$, M. BAXTER CAMPAÑA ${ }^{2}$, \\ M. TOLÓN MARÍN², C. MORALES PÉREZ1', G. FRESNEDA SEPTIEM ${ }^{1}$, \\ M. AVENDAÑO ESPINOSA², F. GUTIÉRREZ GARCÍA ${ }^{1}$ \\ ${ }^{1}$ Departamento de Epidemiología Hospitalaria. Hospital Docente Clínico Quirúrgico \\ "Joaquín Albarrán”. ${ }^{2}$ Centro Provincial de Higiene y Epidemiología. Ciudad de La Habana. \\ Cuba
}

\section{RESUMEN}

Objetivo: Determinar la prevalencia de infección nosocomial (IN) en siete Hospitales Clínico Quirúrgicos de Ciudad de La Habana.

Método: Realizamos un estudio descriptivo de corte transversal de los hospitales universitarios con número de camas superior a 100. La presencia de IN, se determinó mediante la realización de pesquizaje activo en la totalidad de los pacientes ingresados. Se utilizó la técnica estadística de análisis de distribución de frecuencias. Fueron calculadas tasas de IN, por hospital y servicio de atención. También se calculó Odds Ratio puntual y por intervalos.

Resultados: La prevalencia global de IN resultó ser de 9,2 por cada 100 pacientes. Las localizaciones más frecuentes correspondieron a: el sistema cardiovascular (55 casos), el sitio quirúrgico (50 casos) y el tracto urinario (42 casos). El 19,7 \% de los pacientes estudiados, tenían algún dispositivo intravascular colocado. Todos ellos fueron encontrados asociados a la presencia de infección nosocomial. La mayor intensidad resultó ser con la entubación endotraqueal (OR 7.83 IC $95 \%$ 3,32-11,52).

Conclusión: La prevalencia de infecciones nosocomiales en los Hospitales Clínico Quirúrgicos de Ciudad de la Habana hacen necesario fortalecer las actividades de prevención y control, con especial énfasis en su vigilancia.

PALABRAS CLAVE: Infección nosocomial. Prevalencia. Hospitales. Localizaciones. Procedimientos de riesgo.
PREVALENCE OF NOSOCOMIAL INFECTIONS IN UNIVERSITY HOSPITAL OF HAVANA

\begin{abstract}
Objective: Determine nosocomial infection (NI) prevalence in seven University Hospitals of Havana.

Method: A cross sectional survey was carry out in university hospital with more than 100 beds. Presence of NI was determined by an active screening procedure in all patients admited in hospitals. Technical statistics of frequency distribution was used. Rates of NI were estimated for each hospital and in the special case of procedure, Odds Ratio and its confidence interval at $95 \%$ were calculated (CI $95 \%$ ).

Results: Prevalence of NI was $9.2 \%$. The most frequent localization were: cardiovascular system (55 patients), surgical site ( 50 patients) and urinary tract (42 patients ). The $19.7 \%$ of studied patient showed intravascular device. All patients showed NI associated with procedures, the highest intensity was association with an endotracheal tube $($ OR 7.83, IC $95 \% 3.32-11.52$ ).

Conclusion: Nosocomial infections in this hospital is a serious health problem; it is necessary to focus our medical work in this regard for the prevention and control of NI.
\end{abstract}

KEY WORDS: Nosocomial infection. Prevalence. Hospital. Infection types. Risk procedures.

Guanche Garcell H, Núñez Labrador L, Baxter Campaña M, Tolón Marín M, Morales Pérez C, Fresneda Septiem G, Avendaño Espinosa M, Gutiérrez García F. Prevalencia de infección nosocomial en hospitales universitarios de La Habana, Cuba. An Med Interna (Madrid) 2006; 23: 269-271.

\section{INTRODUCCIÓN}

A pesar de que la historia de las infecciones nosocomiales es tan antigua como la historia del hospital, pues existen infecciones hospitalarias desde el momento en que se agrupan los enfermos para su cuidado, aun en la actualidad ellas constituyen una problema de salud relevante, no solo por su morbilidad y mortalidad, sino también por las implicaciones económicas para los sistemas de salud (2-4). Su morbilidad es variable entre diferentes instituciones y naciones, por depender de múltiples factores: número de camas, complejidad de los pacientes y los procederes realizados en ellos. Entre otros factores, estos determinan que las tasas de prevalencia no puedan, en general, ser comparables entre diferentes instituciones $(5,6)$.

En Cuba desde la década del 70 comienzan las primeras actividades de investigación y control de las infecciones hospitalarias. En el año 1973 por la Resolución Ministerial No 51 se definen las funciones del comité de prevención y control de las IN en las unidades hospitalarias. Posteriormente en 1983, estas actividades por su importancia en el Sistema de Salud se integran en el Programa Nacional de Prevención y Control de la IIH (7).

Un componente fundamental en los programas de prevención y control de la infección nosocomial es su vigilancia ruti-

Trabajo aceptado: 17 de enero de 2006 
naria, que en nuestro país se realiza mediante la vigilancia sistemática (método de incidencia). De la misma manera se recomiendan los estudios de prevalencia, fundamentalmente, para monitorear la efectividad de la vigilancia y el control de las infecciones hospitalarias (8).

En nuestro medio, son escasos los reportes de estudios de prevalencia publicados. Este hecho unido a la necesidad de determinar la magnitud del problema en Hospitales universitarios de Ciudad de La Habana, constituyó la principal motivación para realizar el presente estudio.

\section{MATERIAL Y MÉTODO}

Se realizó un estudio de corte transversal para determinar la prevalencia de infección nosocomial (IN) en los hospitales universitarios de La Habana con dotación superior a 100 camas y dedicados a cuidados agudos.

Mediante pesquisa activa se determinó la presencia de IN, en la totalidad de los pacientes ingresados. Fueron excluidos del estudio aquellos ingresados en salas de Psiquiatría. La pesquisa fue realizada por médicos y enfermeras especializados en control de infecciones hospitalarias, considerando los siguientes aspectos: se utilizaron las definiciones de IN del Centro para el Control de Enfermedades (1), pesquisa de cada hospital en un día; con lo cuál cada paciente resultaría incluido solo una vez, utilizar como fuentes de información la historia clínica del paciente (evoluciones médicas y de enfermería, empleo de antibióticos, estudios radiológicos y microbiológicos), así como los reportes verbales del personal que atiende al paciente, y su observación.

De cada paciente se obtuvieron las siguientes variables: servicio de atención (clínico, quirúrgico, terapia), presencia de infección nosocomial, localización de la infección nosocomial, procederes de riesgo realizados (venipuntura, cateterismo centrovenoso, catéter urinario abierto, catéter urinario cerrado y entubación endotraqueal).

Estadística: Fue utilizada la técnica estadística de análisis de distribución de frecuencias. También fueron calculadas las tasas de prevalencia de infección nosocomial (pacientes con IN/total de pacientes ingresados x 100), por grupo de hospitales (clasificados según su dotación de camas) y por "servicios de atención". En el caso particular de los "procederes de riesgo", fue empleado odds ratio y su intervalo de confianza al $95 \%$, para conocer la intensidad de la asociación entre los diferentes procederes de riesgo realizados a los pacientes y la presencia de infección nosocomial.

\section{RESULTADOS}

De forma global, la tasa de infección nosocomial resultó ser de 9,21 casos por cada 100 pacientes hospitalizados. Los hospitales con menos de 200 camas y más de 400, presentaron cifras de prevalencia por encima de la tasa global. De ellos la mayor prevalencia correspondió con el único hospital con 100 a 199 camas incluido en la investigación (14,28 por cada 100 pacientes) (Tabla I).

Las unidades de terapia resultaron ser las de mayor riesgo de IN (16,42 por cada 100 pacientes). A estas, le siguieron los servicios quirúrgicos (12,48 por cada 100 pacientes) y los servicios clínicos $(6,12 \%)$ (Tabla I).

\section{TABLA I}

INFECCIÓN NOSOCOMIAL SEGÚN DOTACIÓN DE CAMAS DE LOS HOSPITALES Y SERVICIOS DE ATENCIÓN. HOSPITALES UNIVERSITARIOS DE LA HABANA, MARZO DEL 2000

\begin{tabular}{llll}
\hline Dotación de camas & $n^{*}$ & No & Tasa** \\
\hline $100-199$ & 119 & 17 & 14,28 \\
$200-299$ & 434 & 34 & 7,83 \\
$300-399$ & 588 & 45 & 7,65 \\
$\geq 400$ & 748 & 78 & 10,43 \\
Total & 1889 & 174 & 9,21 \\
Servicio de atención & & & \\
Clínicos & 1095 & 67 & 6,12 \\
Quirúrgicos & 593 & 74 & 12,48 \\
Terapia & 201 & 33 & 16,42 \\
\hline
\end{tabular}

* Pacientes estudiados.

* Por cada 100 pacientes pesquisados.

En relación con la localización de la IN, la figura 1 muestra que la mayor frecuencia se correspondió con las cardiovasculares (55 casos), seguidas por la sepsis del sitio quirúrgico (50 casos) y las que afectan al tractus urinario (42 casos), y al tractus respiratorio bajo (30 casos), en este mismo orden.

El 19,7\% del total de los pacientes estudiados, tenían algún dispositivo intravascular colocado, siendo la venipuntura el encontrado con mayor frecuencia (16\%). La entubación endotraqueal $(0,8 \%)$ resultó ser el proceder de menor frecuencia. El cateterismo urinario (4,6 y 4,1\%), ocupó posiciones intermedias en relación con los procederes antes mencionados. En general todos ellos fueron encontrados asociados a la presencia de infección nosocomial. La mayor intensidad de la asociación se encontró en el caso de la entubación endotraqueal (OR 7,83; IC 95\% $3,32-11,52)$; en un paciente entubado, el riesgo de infección nosocomial es casi 8 veces mayor que en un paciente que no lo este (Tabla II).

\section{TABLA II}

PROCEDERES DE RIESGO REALIZADOS Y RAZÓN DE ODDS (INTERVALO DE CONFIANZA 95\%) DE INFECCIÓN NOSOCOMIAL, CORRESPONDIENTES. HOSPITALES UNIVERSITARIOS LA HABANA, MARZO DEL 2000

\begin{tabular}{lrrl}
\hline Procederes & $N^{\circ}$ & $\%$ & OR (IC 95 \%) \\
\hline Venipuntura & 302 & 15,99 & $5,9(4,50-7,73)$ \\
Cateterismo centrovenoso & 71 & 3,76 & $5,27(3,82-7,25)$ \\
Cateterismo urinario abierto & 78 & 4,13 & $4,01(2,80-5,73)$ \\
Cateterismo urinario cerrado & 86 & 4,55 & $6,56(4,98-8,65)$ \\
Entubación endotraqueal & 15 & 0,79 & $7,83(3,32-11,52)$ \\
\hline
\end{tabular}

\section{ANÁLISIS DE LOS RESULTADOS}

Entre los propósitos de los estudios de prevalencia puntual aplicados a la vigilancia se encuentra la determinación de la magnitud de las infecciones nosocomiales en instituciones de 


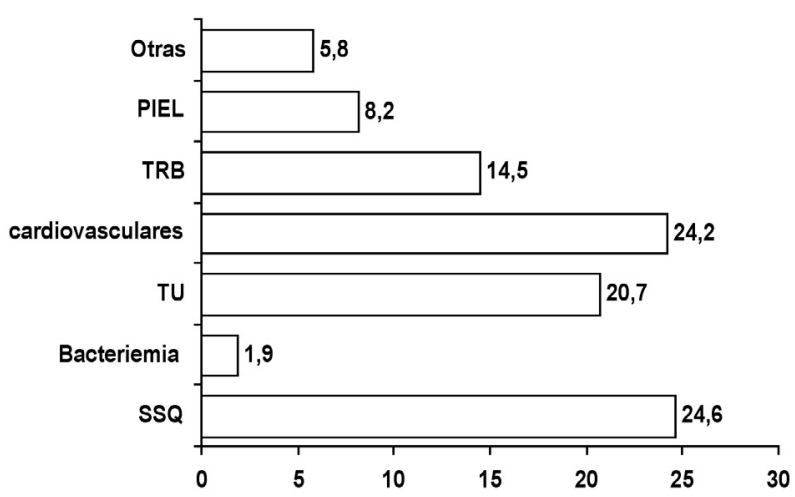

Fig. 1. Distribución proporcional de las infecciones nosocomiales según localización. Hospitales universitarios La Habana, marzo del 2000.

salud (8). El estudio que hemos realizado ha mostrado que en los hospitales universitarios evaluados la prevalencia fue de 9,2 casos/100 pacientes. Otros estudios realizados en instituciones universitarias o de cuidados agudos muestran tasas de prevalencia similares a la demostrada para nuestras institu- ciones, como de $8,5 \%$ en el Libano (9), 9,3\% en hospitales griegos (10), 7\% en hospitales españoles (11), y 11,6\% en hospitales suizos (12).

En la única institución con menos de 200 camas que evaluamos, se observaron las tasas de prevalencia superiores, lo que depende de que la población atendida es predominantemente anciana, se realizan procederes quirúrgicos de mediana complejidad, y es muy frecuente la utilización de dispositivos intravasculares o cateteres urinarios. Estos factores, entre otros, son los que determinan que las tasas de infección sean superiores en las unidades de terapia, cuando se comparan con otros servicios hospitalarios, como ha sido demostrado en múltiples investigaciones sobre el tema $(3,6)$.

Igualmente la frecuencia de utilización de procederes de riesgo determina las localizaciones de infecciones nosocomiales en instituciones de salud. Se ha destacado el empleo de dispositivos intravasculares, procederes quirúrgicos y de cateteres urinarios, y con menor frecuencia de la entubación endotraqueal, entre otros (12-14). Ello determinó la frecuente observación de infecciones arteriales o venosas, del sitio quirúrgico y del tractus urinarios en los hospitales pesquisados.

Este estudio ha hecho evidente que las infecciones nosocomiales en los hospitales universitarios de La Habana constituyen una problema de salud que requiere el fortalecimiento de las actividades de prevención y control, con especial énfasis en su vigilancia sistemática.

\section{Bibliografía}

1. Garner JS, Jarvis WR, Emori TG, Horan TC, Hughes JM. CDC definitions for nosocomial infections. In: Olmsted RN, ed.: APIC Infection Control and Applied Epidemiology: Principles and Practice. St. Louis: Mosby; 1996.

2. Valero LF, Saens MC. Etiología de la infección nosocomial en cirugía: comparación de 2 años (1988 y 1996). Enferm Infecc Microbiol Clin 1998; 16: 79-82.

3. Fridkin SK, Welbel SF, Weinstein RA. Magnitude and prevention of nosocomial infection in the intensive care unit. Infect Dis Clin N Am 1997; 11: 479-496.

4. Weinstein RA. Nosocomial infection update. Emerg Infect Dis 1998; 4 416-20.

5. Hospital Infectious Program. National Center for Infectious Diseases, Centers for Disease Control and Prevention: National Nosocomial Infection Surveillance (NNIS) Report, data summary from October 1986- April 1996, issued May 1996. Am J Infect Control 1996; 24: 380-388.

6. Keita-Perse O, Edwards J, Culver DH, Gaynes RP. Comparing nosocomial infection rates among surgical intensive care units: The importance of separating cardiothoracic and general surgery intensive care-unit. Infect Control Hosp Epidemiol 1998; 19: 260-261.

7. Cuba. Ministerio de Salud Pública. Programa Nacional de Prevención y Control de la Infección Hospitalaria. Ciudad de la Habana. 1996.
8. Gaynes RP. Surveillance of nosocomial infections. En: Bennett JV, Brachman PS, edtitores. Hospital Infection. Philadelphia: LippincottRaven Publishers. 1998.

9. Azzam R, Dramaix M. A one day prevalence survey of hospital acquired infections in Lebanon. J Hosp Infect 2001; 49: 74-8.

10. Gikas A, Pediaditis J, Papadakis JA, Starakis J, Levidiotou S, Nikolaides P, et al. Prevalence study of hospital-acquired infections in 14 Greek hospitals: planning from the local to the national surveillance level. J Hosp Infect 2002; 50: 269-75.

11. Vaque J, Rossello J, Arribas JL Prevalence of nosocomial infections in Spain: EPINE study 1990-1997. EPINE Working Group. J Hosp Infect 1999; 43 (Supl.): S105-11.

12. Harbarth S, Ruef C, Francioli P, Widmer A, Pittet D. Nosocomial infections in Swiss university hospitals: a multi-centre survey and review of the published experience. Swiss-Noso Network. Schweiz Med Wochenschr 1999; 129: 1521-8

13. Nicholls TM, Morris AJ. Nosocomial infection in Auckland Healthcare hospitals. N Z Med J 1997; 110(1050): 314-6

14. Hauer T, Lacour M, Gastmeier P, Schulgen G, Schumacher M, Ruden H, Daschner F. Nosocomial infections intensive care units. A nationwide prevalence study. Anaesthesist 1996; 45: 1184-91 